\title{
Functional salvage of a mangled lower limb using custom-made endoprosthetic replacement
}

\author{
Riazuddin Mohammed · Khalid Baloch • \\ Francis Peart · Adesegun Abudu
}

Received: 29 December 2008/Accepted: 31 August 2009/Published online: 24 September 2009

(C) Springer-Verlag 2009

\begin{abstract}
Functional salvage of a severely injured extremity is a challenge for the patient and the treating surgeon. We report a case of a woman presenting with severely injured lower limb and bone loss, which was managed using a custom-made endoprosthetic replacement for successful functional outcome. Despite being complicated by bone loss, nerve injury and infection; a planned staged surgical treatment and rehabilitation have resulted in satisfactory outcome. At 3-year follow-up, the functional score according to the Musculoskeletal Tumor SocietyInternational Symposium on Limb Salvage System was $70 \%$ and the Toronto Extremity Salvage Score was $62 \%$. Endoprosthetic replacements may have a limited role in managing selected patients with mangled extremity and can lead to a good functional outcome to these patients.
\end{abstract}

Keywords Mangled extremity · Functional salvage · Endoprosthetic replacement

R. Mohammed · K. Baloch

Department of Trauma and Orthopaedics, University Hospital

of Birmingham NHS Trust, Birmingham B29 6JD, UK

F. Peart

Department of Plastics and Reconstructive Surgery,

Royal Orthopaedic Hospital NHS Trust,

Birmingham B31 2AP, UK

A. Abudu

Department of Orthopaedics and Musculoskeletal Oncology, Royal Orthopaedic Hospital NHS Trust,

Birmingham B31 2AP, UK

R. Mohammed ( $\square)$

16 Bayliss Close, Birmingham B31 2XP, UK

e-mail: riaz22@hotmail.co.uk

\section{Introduction}

A severely injured extremity poses difficult decisions for the patient and the treating surgeon. The degree of soft tissue injury, neurovascular damage, bone loss, presence of other injuries, patients' general physical and psychological condition are all important factors in decision-making. The majority of the patients were previously treated by amputation. However, with advances in surgical methods of fracture stabilization, soft tissue reconstruction and microsurgical techniques, some mangled limbs can now be salvaged.

Adequate soft tissue reconstruction is of utmost importance. Various treatment options have been suggested for managing the associated bone loss. These include internal fixation with bone grafting, distraction osteogenesis through circular external fixators; primary shortening followed by staged lengthening and allografts. However, these are associated with very prolonged recovery and the functional outcomes are unpredictable. We report a case of a woman presenting with severely injured lower limb and bone loss which was managed using a custom-made endoprosthetic replacement for successful functional outcome.

\section{Case report}

A 52-year-old woman sustained a severe crushing injury to her left lower limb having been trapped between a wall and a rapidly reversing car. She was conscious, coherent and haemodynamically stable. Her other injuries included a deformed right ankle and some bruising over the right cheek. There was no other major system injury. She was resuscitated according to the advanced trauma life support 
protocol and the bleeding from the leg was attended to. After she was clinically stabilized, a secondary survey was conducted to asses the limb status. Extensive soft tissue injury with degloving of skin and muscle damage from mid-thigh to lower calf was noted.

There was a significant comminution of the lower end of femur with loose fracture fragments visible. Distal pulses were palpable. Neurological assessment was found to be difficult at this stage. The mangled extremity severity score (MESS) was calculated to be 6 ( 3 for high energy injury, 1 for transient hypotension and 2 for age) and was utilised to asses the suitability for limb salvage. There was no significant past medical history or medication and allergy history of note.

The wound was covered with sterile dressings and the limb was splinted. Antibiotic and tetanus prophylaxis were administered. Adequate analgesia was provided. The right ankle and foot were found to be swollen, bruised, but with intact skin and were extremely tender. Trauma series radiographs and limb radiographs confirmed a comminuted distal end left femur fracture with bone loss (Fig. 1), a right ankle bi-malleolar fracture and an undisplaced base of right first metatarsal fracture.

An emergency debridement and stabilization of the femur fracture were obtained using a bridging Hoffman uniaxial external fixator (Fig. 2). The devitalized bone fragments were debrided. Operative findings included loose femur fragments with contamination from fragments of plastic material, partially intact lateral gastrocnemius, partially detached iliotibial band and an avulsed biceps. The knee extensor mechanism and the neurovascular bundle were found to be intact. The right ankle fracture was also internally fixed on the same day.

At this stage, the patient was referred to us due to extensive soft tissue defect and a $12 \mathrm{~cm}$ femur bone loss.

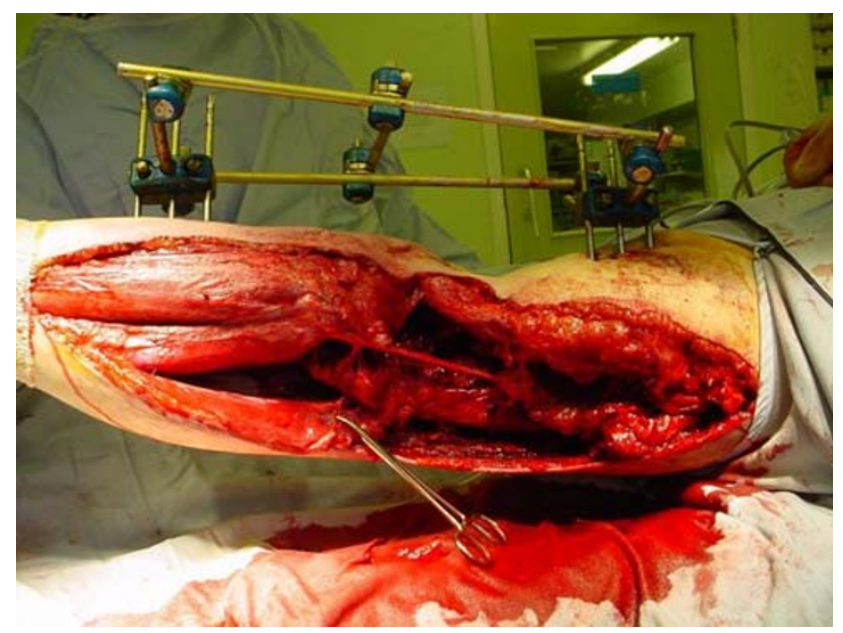

Fig. 1 Soft tissue wound depicting the stretched common peroneal nerve

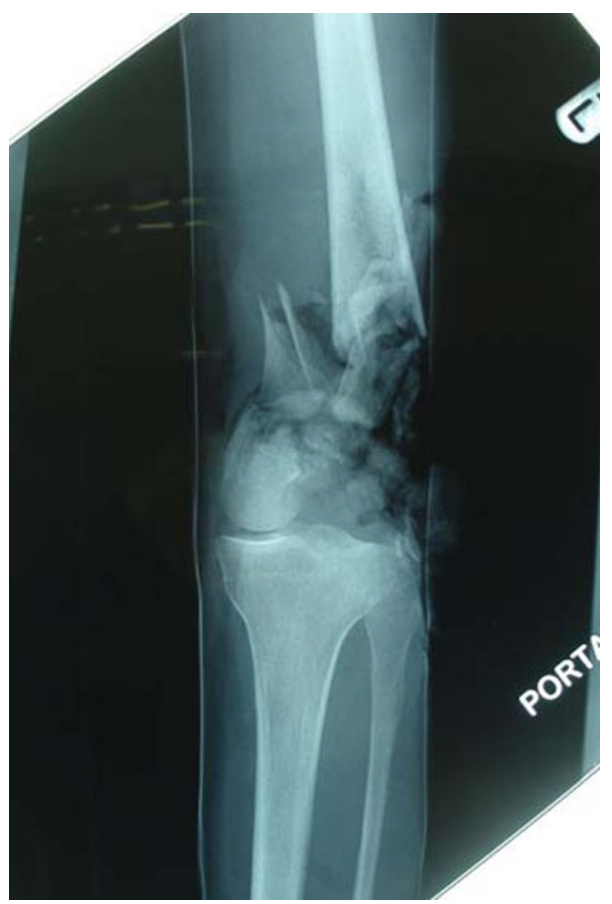

Fig. 2 Plain radiograph confirming a comminuted distal end fracture of left femur with bone loss

The aim was to reconstruct these defects using an endoprosthetic replacement. Option of an amputation and limb preservation was discussed with the patient. The patient preferred limb salvage. The plastic surgeon was also involved in the decision-making process and the patient was informed of the treatment plan. Over the next few days, a relook debridement was done and was followed by repeat debridement. The vascular status of the limb was normal, but there was a foot drop on clinical assessment. The wound exploration revealed that the common peroneal nerve was found to be intact, but had been stretched. A cement spacer to allow for the bone loss and a lateral gastrocnemius flap were harvested with input from the plastic surgeons. Unfortunately, this got infected with bacillus species. The wound was debrided, an across knee intramedullary rod and cement spacer was used to achieve stability. The infection was controlled by intravenous vancomycin.

In view of the ongoing infection and soft tissue problems, it was necessary to pursue on a cautious note. The next few weeks involved multiple wound washouts in the operating theatre to control infection. The wound swabs grew Acinetobacter Baumannii sensitive to the antibiotic merapenem. About 5 weeks after the initial injury, the leg was re-debrided and the cement spacer was revised. A left latismus dorsi free-flap was used for soft tissue reconstruction. The wound was assessed and redressed a few times in the operating theatre subsequently. With continued 
antibiotic treatment for 4 months, the infection was under control and repeated aspirated from the knee were negative.

The leg wound had healed well. However, the knee had only a jog of movement. A distal femur endoprosthetic replacement was planned for about 11 months from the initial injury (Figs. 3, 4). A custom-made distal femoral endoprosthesis, the SMILES implant (Stanmore Modular Individualised Lower Extremity System), Stanmore Implants Worldwide, UK, was used for reconstruction. Quadricepsplasty was needed to achieve sufficient access and the range of movement on table was $0^{\circ}-60^{\circ}$.

The patient underwent staged physiotherapy and gradual rehabilitation. The range of knee movement was of some concern and a manipulation under anaesthesia was performed 6 months after the reconstructive arthroplasty.

\section{Current status}

The patient is now 3 years from the endoprosthetic reconstruction. She is independently mobile and able to drive a car without limitations. The knee flexion remained limited to $0^{\circ}-30^{\circ}$ only. There is no evidence of infection.

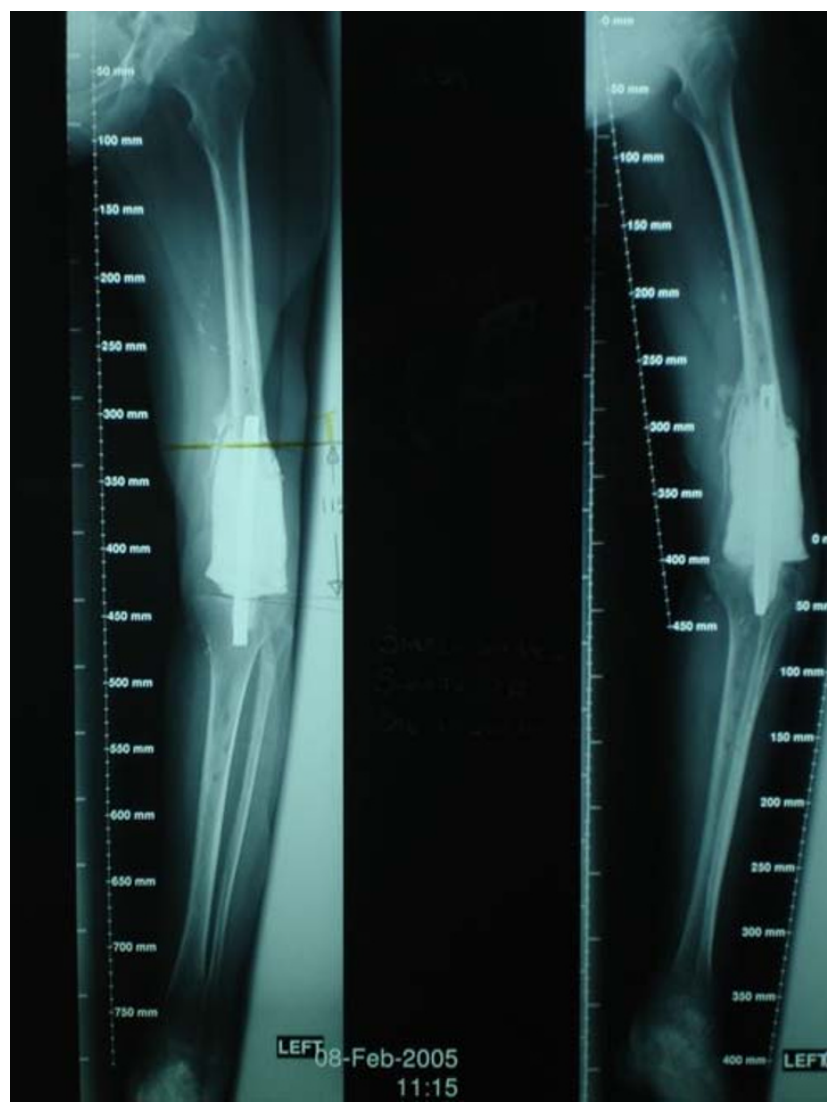

Fig. 3 Long leg radiographs for preoperative planning for the endoprosthetic replacement
The prosthesis remained stable and well cemented. The limb is now $4 \mathrm{~cm}$ short. Functional score according to the Musculoskeletal Tumor Society-International Symposium on Limb Salvage System [1] was 70\% (21 out of a possible 30 points) and the Toronto Extremity Salvage Score [2] was $62 \%$ (93 out of a possible 150 points). Figure 5 shows the latest radiographs at 3 years after surgery.

\section{Discussion}

Mangled limbs with severe bone and soft tissue loss present a clinical challenge in terms of reconstructive surgery, prolonged rehabilitation, physical and psychological demands on the patient, and with no guarantee of a successful outcome. A question of primary amputation or limb reconstruction is a difficult one. Various scoring systems are available to asses the suitability for limb salvage in such situations such as the MESS; the limb salvage index; the predictive salvage index; the nerve injury, ischaemia, soft tissue injury, skeletal injury, shock and the age of patient score; and the Hannover fracture scale-97. Although a recent prospective study questions the clinical usefulness of any of these scores [3], the most commonly



Fig. 4 Manufacturer's proof of the custom-made distal femoral endoprosthesis 




Fig. 5 Plain radiograph of the endoprosthetic replacement at 3 years follow-up

used in the MESS system. Helfet et al. [4] have reported that an MESS score of greater than or equal to $7 \mathrm{had}$ a $100 \%$ predictable value for amputation.

The extents of soft tissue damage and neurovascular status are the important local factors in making the correct initial decision. Hence, significant experience with managing such patients and input from other relevant specialists are essential from the onset.

The goal of limb salvage surgery in such extremity injuries is to provide adequate soft tissue cover, skeletal stabilization, restore adequate length and alignment and most importantly, result in a functioning limb. Although various treatment modalities have been advocated in the management of limb injuries with bone loss, the common element in all these are the multiple reconstructive procedures, long term to recovery and the less than satisfactory functional results. Acute shortening followed by progressive lengthening [5, 6], bone transport using Ilizarov technique [7], allografts to replace bone defects $[8,9]$ and free vascularised bone grafts [10] have all been advocated for the reconstruction of the defects. However, the complications are many and the success limited. The major advantage of cemented endoprosthesis is early stabilization and recovery of function.

We have reconstructed a mangled extremity with $12 \mathrm{~cm}$ bone loss in the femur using a custom-made distal femoral endoprosthetic replacement. To our knowledge, this has so far not been reported in literature. Traditionally, endoprosthetic replacements are used for managing skeletal and soft tissue defects following the tumour resection and have had good long-term results [11]. However, there seem to be additional applications afforded by these systems. Failed internal fixations of fracture, severe fractures with bone defects, failed joint arthroplasty with insufficient bone stock are the non-tumour indications for endoprosthesis' [12]. The advantages with endoprosthetic replacement are the ability to reconstruct massive skeletal and soft tissue defects, ready availability and are relatively inexpensive [13]. The patients can recover rapidly and weight bear early $[14,15]$. The major complications are infection, aseptic loosening, mechanical failure and fracture. Many of the complications can be readily addressed, provided the patient is regularly followed up. Also the surgeon needs to be fully acquainted with the prosthesis and be fully aware of the limitations and dangers of the procedure.

Endoprosthetic replacement for the management of trauma may have a limited, but a defined role in addressing skeletal defects. However, the principles of managing an acute open fracture with bone loss remain the same, whatever the final choice of reconstruction. The initial sequence of management is resuscitation, wound debridement and skeletal stabilization. Appropriate soft tissue cover must be obtained early and any infection be adequately controlled. Only then can secondary osseous reconstruction proceed. This process may take many months, especially if complicated by infection. The limb although having survived the initial trauma can become stiff and functionless. Endoprosthetic replacement with adequate soft tissue procedures can aim to provide a more functional limb that is stable, pain free and has satisfactory range of movements. All through the treatment plan, the patient needs to be fully informed and involved in the process. The treating surgeon and the patient must be able to evaluate the progress, the treatment and change the course of treatment when necessary.

Patients suffer from both physical and emotional consequences following the severe extremity trauma. The goal of limb salvage is to provide a satisfactory limb function at the end of the arduous treatment journey. Endoprosthetic replacements may have a limited role in managing selected patients with mangled extremity and can lead to a good functional outcome to these patients. We recommend that this type of treatment be performed only by specialist teams consisting of surgeons experienced with endoprosthetic surgery, plastic surgeons, specialist microbiologists, wound care nurses, physiotherapists and occupational therapists.

\section{References}

1. Enneking WF, Dunham W, Gebhardt MC, Malawer M, Pritchard DJ (1993) A system for the functional evaluation of 
reconstructive procedures after surgical treatment of tumors of the musculoskeletal system. Clin Orthop 286:241-246

2. Davis AM, Wright JG, Williams JI, Bombardier C, Griffin A, Bell RS (1996) Development of a measure of physical function for patients with bone and soft tissue sarcoma. Qual Life Res 5(5):508-516

3. Bosse MJ, MacKenzie EJ, Kellam JF, Burgess AR, Webb LX, Swiontkowski MF, Sanders RW, Jones AL, McAndrew MP, Patterson BM, McCarthy ML, Cyril JK (2001) A prospective evaluation of the clinical utility of the lower-extremity injuryseverity scores. J Bone Joint Surg [Am] 83-A:3-14

4. Helfet DL, Howey T, Sanders R, Johansen K (1990) Limb salvage versus amputation: preliminary results of the mangled extremity severity score. Clin Orthop Relat Res 256:80-86

5. Lerner A, Fodo L, Soudry M, Peled IJ, Herer D, Ullmann Y (2004) Acute shortening: modular treatment modality for severe combined bone and soft tissue loss of the extremities. J Trauma 57(3):603-608

6. Saleh M, Rees A (1995) Bifocal surgery for deformity and bone loss after lower-limb fractures: comparison of bone-transport and compression-distraction methods. J Bone Joint Surg $[\mathrm{Br}]$ 77-B:429-434

7. Mekhail A, Abraham E, Gruber B, Gonzalez M (2004) Bone transport in the management of posttraumatic bone defects in the lower extremity. J Trauma 56(2):368-378
8. Beaver RJ, Mahomed M, Backstein D, Davis A, Zukor DJ, Gross AE (1992) Fresh osteochondral allografts for post-traumatic defects in the knee: a survivorship analysis. J Bone Joint Surg [Br] 74-B:105-110

9. Salai M, Horoszowski H, Pritsch M, Amit Y (1999) Primary reconstruction of traumatic bony defects using allografts. Arch Orthop Trauma Surg 119:435-439

10. Allende C, Allende BT (2004) Posttraumatic one-bone forearm reconstruction: a report of seven cases. J Bone Joint Surg [Am] 86-A:364-369

11. Myers GJ, Abudu AT, Carter SR, Tillman RM, Grimer RJ (2007) Endoprosthetic replacement of the distal femur for bone tumours: long-term results. J Bone Joint Surg [Br] 89(4):521-526

12. Freedman EL, Eckardt JJ (1997) A modular endoprosthetic system for tumor and non-tumor reconstruction: preliminary experience. Orthopedics 20(1):27-36

13. Grimer RJ, Carter SR, Tillman RM, Sneath RS, Walker PS, Unwin PS, Shewell PC (1999) Endoprosthetic replacement of the proximal tibia. J Bone Joint Surg [Br] 81(3):488-494

14. Unwin PS, Cobb JP, Walker PS (1993) Distal femoral arthroplasty using custom-made prostheses: the first 218 cases. J Arthroplasty 8(3):259-268

15. Bradish CF, Kemp HB, Scales JT, Wilson JN (1987) Distal femoral replacement by custom-made prostheses: clinical follow-up and survivorship analysis. J Bone Joint Surg [Br] 69(2):276-284 